\title{
Editorial
}

\section{Real-Time Communication in Wireless Sensor Networks}

\author{
Jeongcheol Lee, ${ }^{1}$ Babar Shah $\mathbb{D},{ }^{2}$ Giovanni Pau $\mathbb{D}^{3},{ }^{3}$ Javier Prieto $\mathbb{D}^{4},{ }^{4}$ and Ki-Il Kim $\mathbb{D}^{5}$ \\ ${ }^{1}$ Department of Scientific Platform Development, Computational Science \& Engineering Center, \\ Korea Institute of Science and Technology Information (KISTI), Daejeon, Republic of Korea \\ ${ }^{2}$ College of Technological Innovation, Zayed University, Abu Dhabi, UAE \\ ${ }^{3}$ Kore University of Enna, Enna, Italy \\ ${ }^{4}$ BISITE Research Group, University of Salamanca, Salamanca, Spain \\ ${ }^{5}$ Department of Computer Science and Engineering, Chungnam National University, Daejeon, Republic of Korea \\ Correspondence should be addressed to Ki-Il Kim; kikim@cnu.ac.kr
}

Received 9 January 2018; Accepted 11 January 2018; Published 8 February 2018

Copyright (C) 2018 Jeongcheol Lee et al. This is an open access article distributed under the Creative Commons Attribution License, which permits unrestricted use, distribution, and reproduction in any medium, provided the original work is properly cited.

Through wireless sensor networks (WSN), we can acquire the various interesting event information around sensor nodes through multihop communications. In WSN, there are two types of applications, that is, event or query based. Commonly, in these application, the value on each sensor node is very sensitive to delay or latency. So, it is strongly required to deliver data to sink node within the deadline since data received after the deadline is not acceptable at all in WSN. The good example of application demanding real-time communication in WSN includes tracking of moving object and intrusion detection.

However, compared to typical networks, it is very difficult to achieve real-time communication in WSN. Severe constraints such as limited computing power and narrow bandwidth are not suitable to provide real-time communication accordingly. So, a number of important issues and research challenges have to be addressed to provide realtime communication in WSN. Based on this demand, this special issue is planned to contribute to advances in real-time communications in WSN.

While considering our objective, editors believe that this special issue provides collection of articles on networking technique in real-time communications. We have selected 4 valuable papers by evaluating several aspects such as relevance to special issue and novelty of solution. The topic of these papers is roughly categorized into the following areas: multichannel transmission, MAC protocol, testbed for routing protocol, and comprehensive survey for real-time in WSN.
In the paper entitled "Priority-Based Dynamic Multichannel Transmission Scheme for Industrial Wireless Networks," Y. Igarashi et al. proposed priority-based dynamic multichannel transmission scheme for industrial wireless sensor networks (IWSN) where applications are required to provide precise measurement functions as feedback for controlling devices. In order to guarantee latency for unpredictable on-demand communications, a root node controls the transmission timing of high-priority packets, while other nodes autonomously decide what channel to use and when to transmit packets to a neighbor. In the proposed scheme, packet priority is determined in accordance with application requirements. The proposed scheme operates over a MAC layer and does not rely on any specific MAC protocol. Since there are some standard protocols for real-time communications in IWSN such as ISA100.11a and wirelessHART, the authors discuss compatibility with ISA100.11a in this paper.

Another paper is related to MAC protocol. However, instead of general MAC protocol, T. Kim et al. proposed an efficient MAC protocol for radio frequency (RF) energy harvesting in WSN, called REACH. Unlike conventional RF energy harvesting methods, an Energy Transmitter (ET) in the proposed scheme can actively send RF energy signals without Request-for-Energy (RFE) messages. An ET determines the active energy signal transmission according to the consequence of the passive energy harvesting procedures. The other feature of the proposed scheme is that an ET participates contention-based channel access procedure. The simulation results reveal that the proposed protocol can 
increase the energy harvesting rate and the lifetime of WSN by the help of the active energy signal transmission method.

In addition to approaches in physical and data link, $\mathrm{W}$. Jiang et al. addressed the testbed for multipath routing protocol to manage deployment cost. To achieve this goal, a reconfigurable testbed, which supports dynamic protocol switching by creating a novel architecture and experiments with several different protocols, is presented. Based on architecture to separate control and data plane in this testbed, independent routing configuration and data transmission are assumed. Moreover, a programmable flow table provides the testbed with the ability to switch protocols dynamically. The experiments demonstrate that the testbed can reduce the complexity of network management and updating by separating the control and data planes. A user can manage the network easily by simply configuring different behaviors, without redeploying the whole network. Therefore, this reconfiguration function can support WSN virtualization when the scale is growing up and can be used in a multitenant architecture for cloud service.

Another paper is a comprehensive survey paper for literature reviews in real-time communications in WSN. Even though few survey literatures were published, they are outof-date and fail to provide overview of diverse protocols. To address this problem, this survey paper is likely to collect the research work since 2010 and include the potential real-time application and applicable platform for real-time communication in WSN. In addition, unlike previous survey papers, B.-S. Kim et al. included firm real-time model with hard and soft real-time model in WSN. Since several research works for $(m, k)$-firm model in WSN have been proposed recently, it is recommended to analyze and compare them accordingly. Related to rule for categorization, research area on layered architecture has been chosen. In each category, recent research work is described and explained. Finally, the open issues and further research challenges are discussed.

\section{Acknowledgments}

Finally, we appreciate all authors, reviewers, and editorial members for their invaluable contribution. Without their hard work and dedication, it would not have been possible to select these highly qualified papers within the given time limits of this special issue.

Jeongcheol Lee Babar Shah

Giovanni Pau

Javier Prieto

$\mathrm{Ki}$-Il Kim 


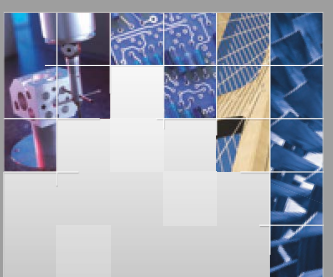

\section{Enfincering}
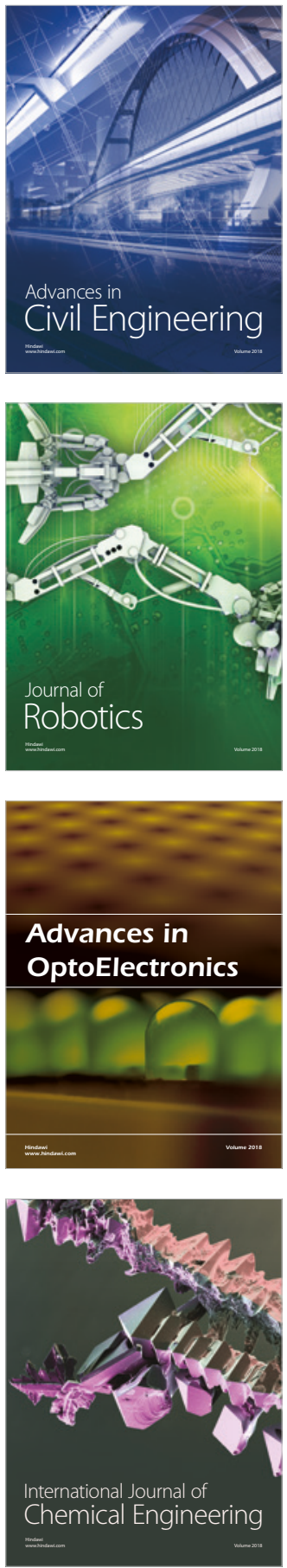

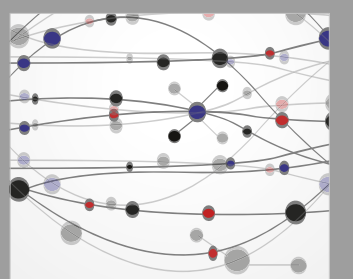

\section{Rotating \\ Machinery}

The Scientific World Journal

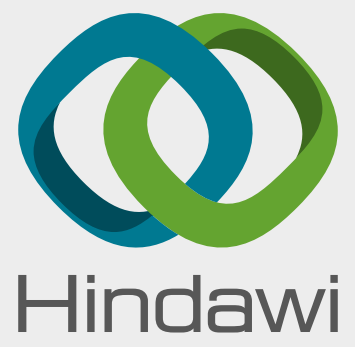

Submit your manuscripts at

www.hindawi.com
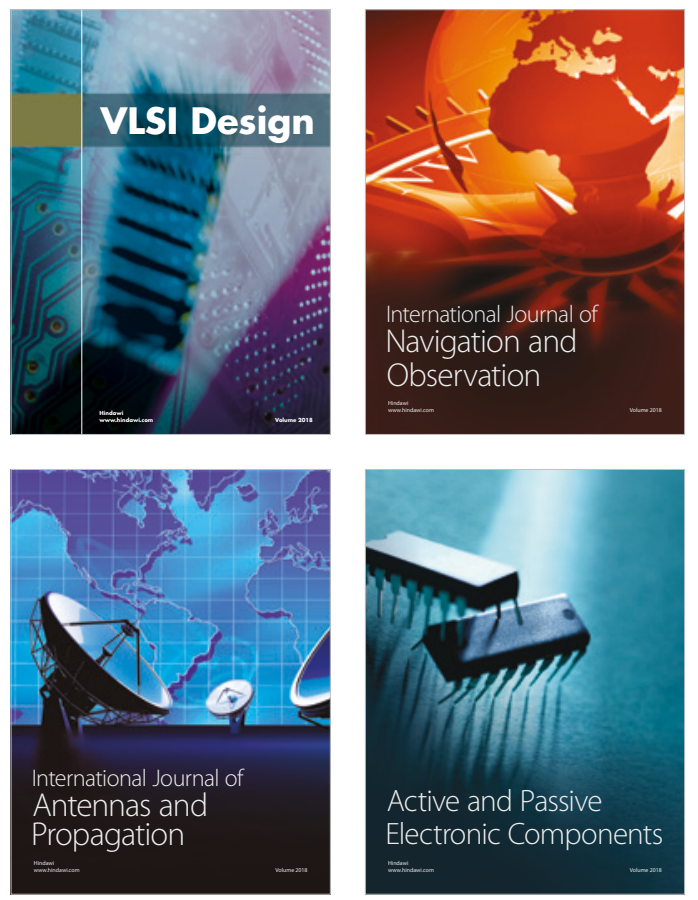
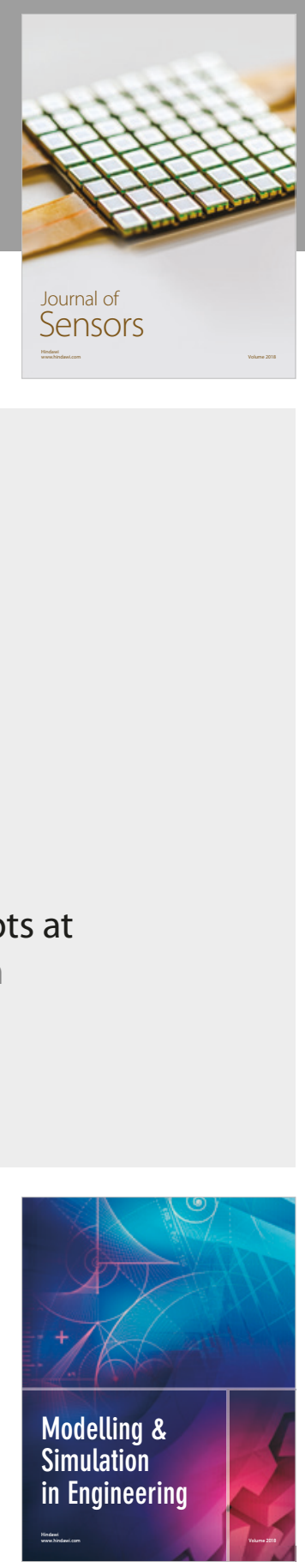

\section{Advances \\ Multimedia}
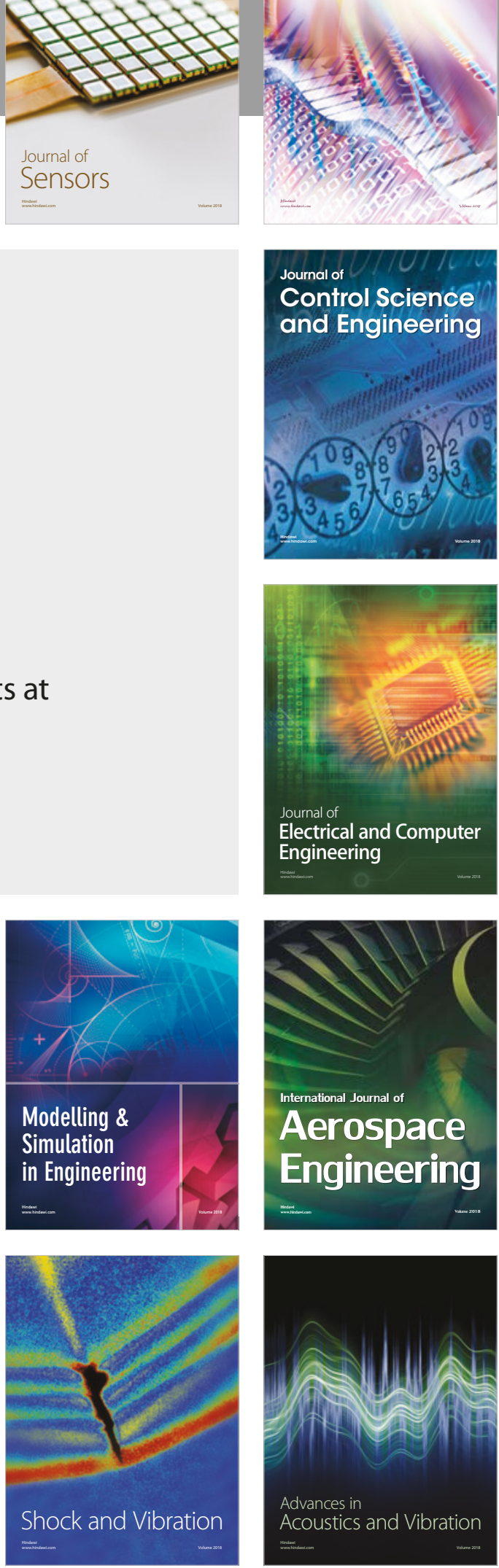